\title{
Decomposition Of Permittivity Contributions From Reflectance Using Mechanism Models
}

\author{
H.T. Banks ${ }^{1}$, Jared Catenacci ${ }^{2}$, Shuhua $\mathrm{Hu}^{3}$, and Zackary R. Kenz ${ }^{4}$
}

\begin{abstract}
In this paper, we investigate the properties of a complex nonmagnetic material through the reflectance, where the permittivity is described by a mechanism model in which an unknown probability measure is placed on the model parameters. Specifically, we consider whether or not this unknown probability measure can be determined from the reflectance or the derivatives of the reflectance, and we also investigate the effect of measurement noise on the estimation. The numerical results demonstrate that if only the reflectance can be observed, then the distribution form cannot be recovered even in the case where the measurement noise level is small. However, if both the reflectance and the derivative of the reflectance can be observed, then the estimated distribution is reasonably close to the true one even in the case where the measurement noise level is relatively high.
\end{abstract}

\section{INTRODUCTION}

The decomposition of a material's electromagnetic response into the elementary component mechanisms responsible for observed phenomena is a fundamental problem of spectroscopy. In the setting of nonmagnetic materials, this involves determining the components of the permittivity using the measured spectral responses. Typically one would assume a particular combination of polarization models (Debye, Lorentz, Gaussian, etc.) with a predetermined number of dielectric parameters. However, in practice, the type of polarization model and the number of constituent dielectric mechanisms are usually unknown. In addition, the resulting decomposition may be non-unique or even nonphysical by using the reflectance (the ready observable in the primary experiment) alone.

Our approach in this work involves imposing a probability measure on model parameters as there are now incontrovertible experimental arguments for distributions of relaxation times for complex materials (e.g., see [5] and the references therein). We then develop a computational framework to nonparametrically estimate the probability measure, and investigate what type of

This research was supported in part by Grant Number NIAID R01AI071915-10 from the National Institute of Allergy and Infectious Diseases, in part by the Air Force Office of Scientific Research under grant number AFOSR FA9550-12-1-0188, and in part by the National Science Foundation under Research Training Grant (RTG) DMS-0636590.

The authors are with Center for Research in Scientific Computation, North Carolina State University, Raleigh, NC 27695-8205, USA

1 htbanksancsu.edu 2 jwcatenalncsu.edu

3 shu3@ncsu.edu 4 zrkenzlncsu.edu measurement information is needed to accurately estimate the probability measure. We remark that some interferometers have the capability to obtain the derivative of the reflectance. Specifically, the Bruker $80 \mathrm{~V}$ two beam interferometer [10, pp. 134-135] is capable of calculating derivatives up to the fifth order using the Savitzky-Golay algorithm. This algorithm smoothes the data through fitting successive sub-sets of adjacent data points with a low-degree polynomial (e.g., see Wikipedia, [14] and the references therein for details). Hence, in this work, we will consider two types of measurements, one corresponding to the reflectance, and the other corresponding to both the reflectance and the derivative the reflectance.

\section{PROBLEM SETUP}

For simplicity, we assume that a monochromatic uniform wave of frequency $\omega$ is incident at an angle $\theta$ on a plane interface between free space and a nonmagnetic dielectric medium as depicted schematically in Figure 1. This medium is assumed to be linear, homogeneous and isotropic. We first describe the reflection coefficient for the case where the wave is incident on a plane interface between two lossy media and then simplify to the particular situation of interest here.

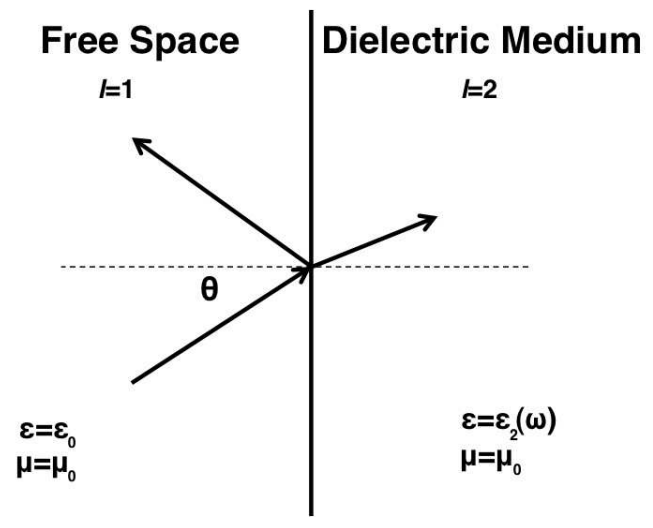

Fig. 1. A monochromatic uniform wave is incident at an angle $\theta$ on a plane interface between a free space and a nonmagnetic dielectric medium, where $\omega$ denotes the frequency of the wave. 


\section{A. General Reflection Coefficient, Interface Between Two Lossy Materials}

In this section, we give details for the reflection coefficient in the case of a uniform plane wave that is incident at an arbitrary angle $\theta$ on a plane interface between two lossy media (which are assumed to be linear, homogeneous and isotropic), where the permittivity, permeability and conductivity of medium $l$ are denoted by $\widehat{\varepsilon}_{l}, \widehat{\mu}_{l}$, and $\widehat{\sigma}_{l}$, respectively. We remark that the arguments to derive the reflection coefficient for this case are similar to those in [12, Section 9.3]. Hence, we only sketch the ideas.

We assume that the electric field is polarized perpendicular to the plane of incidence (TE polarization). Then the incident electric field and incident magnetic field phasors are respectively given by

$$
\begin{aligned}
\widetilde{\mathbf{E}}^{i} & =\widetilde{\mathbf{E}}_{0}^{i} \exp \left(-\left(\gamma_{1} \mathbf{d}_{i} \cdot \mathbf{r}\right),\right. \\
\widetilde{\mathbf{H}}^{i} & =\frac{\mathbf{d}^{i} \times \widetilde{\mathbf{E}}_{0}^{i}}{\eta_{c 1}} \exp \left(-\gamma_{1} \mathbf{d}_{i} \cdot \mathbf{r}\right) .
\end{aligned}
$$

In the above equation, $\mathbf{r}=(x, y, z)^{T}, \mathbf{d}_{i}=$ $(\sin (\theta), 0, \cos (\theta))^{T}$ denotes the unit vector for the direction of the incident wave, and $\widetilde{\mathbf{E}}_{0}^{i}=\left(0, \widetilde{E}_{0}^{i}, 0\right)^{T}$ with $\widetilde{E}_{0}^{i}$ being the incident intensity. In addition, $\gamma_{l}$ represents the propagation constant in medium $l$, and $\eta_{c l}$ denotes the complex intrinsic impedance in medium $l$. They are respectively given by

$$
\gamma_{l}=i \omega \sqrt{\widehat{\varepsilon}_{c l}(\omega) \widehat{\mu}_{l}(\omega)}, \quad \eta_{c l}=\sqrt{\frac{\widehat{\mu}_{l}(\omega)}{\widehat{\varepsilon}_{c l}(\omega)}}, \quad l=1,2,
$$

where $i$ denotes the imaginary unit, and $\widehat{\varepsilon}_{c l}$ represents the complex permittivity of medium $l$, and is given by

$$
\widehat{\varepsilon}_{c l}(\omega)=\widehat{\varepsilon}_{l}(\omega)-i \frac{\widehat{\sigma}_{l}(\omega)}{\omega} .
$$

The reflected electric field and the reflected magnetic field are then given by

$$
\begin{aligned}
& \widetilde{\mathbf{E}}^{r}=\widetilde{\mathbf{E}}_{0}^{r} \exp \left(-\boldsymbol{\Gamma}^{r} \cdot \mathbf{r}\right)=\widetilde{\mathbf{E}}_{0}^{r} \exp \left(-\left(\Gamma_{x}^{r} x+\Gamma_{z}^{r} z\right)\right), \\
& \widetilde{\mathbf{H}}^{r}=\frac{1}{\omega \widehat{\mu}_{1}(\omega)}\left(-i \boldsymbol{\Gamma}^{r} \times \widetilde{\mathbf{E}}_{0}^{r}\right) \exp \left(-\left(\Gamma_{x}^{r} x+\Gamma_{z}^{r} z\right)\right),
\end{aligned}
$$

where $\widetilde{\mathbf{E}}_{0}^{r}=\left(0, \widetilde{E}_{0}^{r}, 0\right)^{T}$ with $\widetilde{E}_{0}^{r}$ being the reflected intensity, and $\Gamma^{r}$ denotes the propagation vector of the reflected field and is given by $\Gamma^{r}=\left(\Gamma_{x}^{r}, 0, \Gamma_{z}^{r}\right)^{T}$ with $\left(\Gamma_{x}^{r}\right)^{2}+\left(\Gamma_{z}^{r}\right)^{2}=\gamma_{1}^{2}$. In addition, the transmitted fields can be written as

$$
\begin{aligned}
\widetilde{\mathbf{E}}^{t} & =\widetilde{\mathbf{E}}_{0}^{t} \exp \left(-\boldsymbol{\Gamma}^{t} \cdot \mathbf{r}\right)=\widetilde{\mathbf{E}}_{0}^{t} \exp \left(-\left(\Gamma_{x}^{t} x+\Gamma_{z}^{t} z\right)\right), \\
\widetilde{\mathbf{H}}^{t} & =\frac{1}{\omega \widehat{\mu}_{2}(\omega)}\left(-i \boldsymbol{\Gamma}^{t} \times \widetilde{\mathbf{E}}_{0}^{t}\right) \exp \left(-\left(\Gamma_{x}^{t} x+\Gamma_{z}^{t} z\right)\right),
\end{aligned}
$$

where $\widetilde{\mathbf{E}}_{0}^{t}=\left(0, \widetilde{E}_{0}^{t}, 0\right)^{T}$ with $\widetilde{E}_{0}^{t}$ being the transmitted intensity, and $\boldsymbol{\Gamma}^{t}$ denotes the propagation vector of transmitted field and is given by $\Gamma^{t}=\left(\Gamma_{x}^{t}, 0, \Gamma_{z}^{t}\right)^{T}$ with $\left(\Gamma_{x}^{t}\right)^{2}+\left(\Gamma_{z}^{t}\right)^{2}=\gamma_{2}^{2}$.
Since the tangential components of both the electric field and the magnetic field are continuous on the interface, we can show that the angle of reflection is equal to the angle of incidence (the law of reflection), and that

$$
\begin{aligned}
& \widetilde{E}_{0}^{i}+\widetilde{E}_{0}^{r}=\widetilde{E}_{0}^{t}, \\
& r_{1 s}\left(\widetilde{E}_{0}^{r}-\widetilde{E}_{0}^{i}\right)=-r_{2 s} \widetilde{E}_{0}^{t},
\end{aligned}
$$

where $r_{1 s}$ and $r_{2 s}$ are given by

$$
\begin{aligned}
& r_{1 s}=\frac{\sqrt{\widehat{\varepsilon}_{c 1}(\omega)} \cos (\theta)}{\sqrt{\widehat{\mu}_{1}(\omega)}} \\
& r_{2 s}=\frac{1}{\widehat{\mu}_{2}(\omega)} \sqrt{\widehat{\varepsilon}_{c 2}(\omega) \widehat{\mu}_{2}(\omega)-\widehat{\varepsilon}_{c 1}(\omega) \widehat{\mu}_{1}(\omega) \sin ^{2}(\theta)} .
\end{aligned}
$$

Note that the reflection coefficient $r_{s}$ is the ratio of reflected intensity $\widetilde{E}_{0}^{r}$ to the incident intensity $\widetilde{E}_{0}^{i}$, Hence, by solving (II.2) we find

$$
r_{s}(\omega, \theta)=\frac{r_{1 s}-r_{2 s}}{r_{1 s}+r_{2 s}} .
$$

Note that the reflection coefficient $r_{s}$ is dependent on frequency, and in general is complex. Next we turn our attention to simplifying the expression for the reflection coefficient to match the set up of the problem of interest.

\section{B. Reflection Coefficient, Interface Between Free Space} and a Lossy Material

In free space, $\widehat{\mu}_{1} \equiv \mu_{0}$ and $\widehat{\varepsilon}_{c l} \equiv \varepsilon_{0}$, where $\varepsilon_{0}$, $\mu_{0}$ are, respectively, the permittivity and permeability in free space. Also, since our material is nonmagnetic, we have that $\widehat{\mu}_{2} \equiv \mu_{0}$. Note that the conductivity in the dielectric material is very small. Hence, we assume that it is negligible, that is, $\widehat{\sigma} \equiv 0$. Thus, by (II.1) we have $\widehat{\varepsilon}_{c 2}(\omega)=\widehat{\varepsilon}_{2}(\omega)$.

Once we make the requisite substitutions in (II.3) and (II.4) we obtain

$$
\begin{aligned}
r_{1 s} & =\sqrt{\frac{\varepsilon_{0}}{\mu_{0}}} \cos (\theta), \\
r_{2 s} & =\frac{1}{\mu_{0}} \sqrt{\widehat{\varepsilon}_{2}(\omega) \mu_{0}-\varepsilon_{0} \mu_{0} \sin ^{2}(\theta)} \\
& =\frac{1}{\sqrt{\mu_{0}}} \sqrt{\widehat{\varepsilon}_{2}(\omega)-\varepsilon_{0} \sin ^{2}(\theta)},
\end{aligned}
$$

and so the reflection coefficient for the given scenario is

$$
\begin{aligned}
r_{s}(\omega, \theta) & =\frac{\sqrt{\varepsilon_{0}} \cos (\theta)-\sqrt{\widehat{\varepsilon}_{2}(\omega)-\varepsilon_{0} \sin ^{2}(\theta)}}{\sqrt{\varepsilon_{0}} \cos (\theta)+\sqrt{\widehat{\varepsilon}_{2}(\omega)-\varepsilon_{0} \sin ^{2}(\theta)}} \\
& =\frac{\cos (\theta)-\sqrt{\widehat{\varepsilon}_{r 2}(\omega)-\sin ^{2}(\theta)}}{\cos (\theta)+\sqrt{\widehat{\varepsilon}_{r 2}(\omega)-\sin ^{2}(\theta)}},
\end{aligned}
$$

where $\widehat{\varepsilon}_{r 2}$ is the relative permittivity of medium 2 and is given by $\widehat{\varepsilon}_{2} / \varepsilon_{0}$. We remark that the above equation is equivalent to Fresnel's Equation with the exception that in (II.6) we have explicitly incorporated frequency 
dependence into the material parameter $\widehat{\varepsilon}_{2}$. Finally we note that (II.6) will serve as the primary focus of our investigation.

\section{Composition of Permittivity}

Without loss of generality, we assume that the permittivity of the dielectric medium is described by a Lorentz model where a probability measure is imposed on the relaxation times. The Lorentz model for electronic polarization represents an oscillating restoring response due to an electric field. The relative permittivity of a single-resonance Lorentz model has the form

$$
\widehat{\varepsilon}_{r 2}(\omega)=\varepsilon_{\infty}-\frac{\omega_{p}^{2}}{\omega^{2}-i \omega / \tau-\omega_{0}^{2}} .
$$

In the above equation, $\varepsilon_{\infty}$ denotes the relative permittivity of the medium at infinite frequency, $\tau$ is the relaxation time, and $\omega_{p}=\omega_{0} \sqrt{\varepsilon_{s}-\varepsilon_{\infty}}$ is called the plasma frequency of the medium, where $\omega_{0}$ is the resonance frequency, and $\varepsilon_{s}$ is the relative permittivity of the medium at zero frequency.

To allow for a distribution $F$ of relaxation times over an admissible set $\mathcal{T} \subset \mathbb{R}$, we generalize the relative permittivity to be

$$
\widehat{\varepsilon}_{r 2}(\omega ; F)=\varepsilon_{\infty}-\int_{\mathcal{T}} \frac{\omega_{p}^{2}}{\omega^{2}-i \omega / \tau-\omega_{0}^{2}} d F(\tau),
$$

where $F \in \mathcal{P}(\mathcal{T})$, the set of admissible probability measures on $\mathcal{T}$. Then the distribution-dependent reflection coefficient is given by

$$
r_{s}(\omega, \theta ; F)=\frac{\cos (\theta)-\sqrt{\widehat{\varepsilon}_{r 2}(\omega ; F)-\sin ^{2}(\theta)}}{\cos (\theta)+\sqrt{\widehat{\varepsilon}_{r 2}(\omega ; F)-\sin ^{2}(\theta)}} .
$$

It is worth noting that this approach of imposing a probability measure on the model parameters was also considered in [5], [11], where the authors directly simulate Maxwell's equations, attempting to estimate a distribution on relaxation times for a Debye medium.

\section{COMPUTATIONAL FRAMEWORK}

In this section we examine an inverse problem methodology for estimating the probability measure of relaxation times with simulated data at various noise levels. In these problems the observable considered is either the magnitude of the reflection coefficient or both the magnitude of the reflection coefficient and its derivatives. For both cases, we assume that the incident angle $\theta$ is zero.

\section{A. Statistical Model}

We consider a statistical model of the form

$$
Y_{j}=h\left(\omega_{j} ; F_{0}\right)+\mathcal{V}_{j}, \quad j=0,1,2,3, \ldots, n .
$$

In the above equation, $h\left(\omega_{j} ; F_{0}\right)$ corresponds to the observed part of the system with the "true" probability measure $F_{0}$ at the measurement frequency $\omega_{j}$, $\mathcal{V}_{j}$ denotes the measurement error at the measurement frequency $\omega_{j}$, and $n+1$ is the total number of observations. For the current proof of concept discussion, we assume that $\mathcal{V}_{j}, j=1,2,3, \ldots, n$, are independent and identically distributed with zero mean and constant covariance matrix $\Sigma_{0}=\sigma_{0}^{2} \mathbf{I}_{\kappa}$, where $\mathbf{I}_{\kappa}$ is a $\kappa \times \kappa$ identity matrix with $\kappa$ being the dimension of $\mathcal{V}_{j}$.

As discussed above, we consider two types of data. In the case that the observed part of the system is the magnitude of the reflection coefficient (or reflectance), we have

$$
h\left(\omega_{j} ; F\right)=\left|r_{s}\left(\omega_{j}, 0 ; F\right)\right| .
$$

For the case in which the data contains both the reflectance and the derivative of the reflectance, $h$ has two components (i.e., $\kappa=2$ ) and is given by

$h\left(\omega_{j} ; F\right)=\left(\left|r_{s}\left(\omega_{j}, 0 ; F\right)\right|, \frac{\frac{\partial}{\partial \omega}\left|r_{s}\left(\omega_{j}, 0 ; F\right)\right|}{\max _{j} \frac{\partial}{\partial \omega}\left|r_{s}\left(\omega_{j}, 0 ; F\right)\right|}\right)^{T}$.

We remark that the reason for choosing the second component of $h$ as above is because the magnitude of the derivative of the reflectance is too small (for the parameter values that we choose for our proof of concept computations) compared to the value of the reflectance.

\section{B. Inverse Problem}

Under the assumptions for the measurement errors in the statistical model, the estimate $\widehat{F}$ of $F$ can be obtained using the ordinary least squares formulation (e.g., see [8] for details)

$$
\widehat{F}=\operatorname{argmin}_{F \in \mathcal{P}(\mathcal{T})} J(F),
$$

where the cost functional $J$ is defined as

$$
J(F)=\sum_{j=0}^{n}\left(h\left(\omega_{j} ; F\right)-y_{j}\right)^{T}\left(h\left(\omega_{j} ; F\right)-y_{j}\right),
$$

and $y_{j}$ is a realization of $Y_{j}$. The existence of $\widehat{F}$ can be established under the Prohorov Metric Framework as developed in [1], [2], [4], [6], [5], [7]. Specifically, if we assume that $\mathcal{T}$ is compact, then it is well known that $\mathcal{P}(\mathcal{T})$ is a compact metric space when taken with the Prohorov [13] metric $\rho^{*}$ (e.g., see [1], [2], [6] for details). Note that $h$ is continuous with respect to $F$. Hence, $J$ is continuous with respect to $F$. Thus, we know that there exists a solution to the least squares problem (III.4).

We note that (III.4) is an infinite dimensional optimization problem (as $\mathcal{P}(\mathcal{T})$ is an infinite dimensional space). Hence, we need to approximate the infinite dimensional space $\mathcal{P}(\mathcal{T})$ with finite dimensional space $\mathcal{P}^{N}(\mathcal{T})$ so that we have a computational tractable finite- 
dimensional optimization problem given by

$$
\widehat{F}^{N}=\operatorname{argmin}_{F \in \mathcal{P}^{N}(\mathcal{T})} J(F) .
$$

Of course, one needs to choose $\mathcal{P}^{N}(\mathcal{T})$ such that $\mathcal{P}^{N}(\mathcal{T}) \rightarrow \mathcal{P}(\mathcal{T})$ in some sense as $N \rightarrow \infty$ so that $\widehat{F}^{N}$ approaches to the solution to (III.4) as $N$ goes to infinity. One such approximation approach involves the use of Dirac delta measures to approximate probability measures $F$; that is,

$$
F \approx F^{N}=\sum_{k=1}^{N} \alpha_{k} \Delta_{\tau_{k}}
$$

where the weights $\alpha_{k}, k=1,2, \ldots, N$, are non-negative real numbers such that $\sum_{k=1}^{N} \alpha_{k}=1$, and $\Delta_{\tau_{k}}$ is a Dirac delta measure with atom at node $\tau_{k}$. With this approximation, the least squares problem that we wish to solve is (III.6) with

$$
\begin{array}{r}
\mathcal{P}^{N}(\mathcal{T})=\left\{F \in \mathcal{P}(\mathcal{T}) \mid F=\sum_{k=1}^{N} \alpha_{k} \Delta_{\tau_{k}},\right. \\
\left.\sum_{k=1}^{N} \alpha_{k}=1\right\}
\end{array}
$$

Under this computational framework the optimization problem (III.6) is reduced to a standard optimization problem over $\mathbb{R}^{N}$ in which we seek to estimate the finite set of values $\left\{\alpha_{k}\right\}_{k=1}^{N}$. The theoretic foundation for such approximation relies on the Prohorov Metric Framework. Specifically, it was shown in [2] that if $\mathcal{T}^{\infty}=\left\{\tau_{k}\right\}_{k=1}^{\infty}$ is an enumeration of the rational numbers of $\mathcal{T}$, then for any element $F \in \mathcal{P}(\mathcal{T})$ there exists a sequence $\left\{F^{N_{j}}\right\}$ with $F^{N_{j}} \in \mathcal{P}^{N_{j}}\left(\mathcal{T}^{\infty}\right)$ such that $\rho^{*}\left(F^{N_{j}}, F\right) \rightarrow 0$ as $N_{j} \rightarrow \infty$. Thus, we see that this delta approximation method can be used regardless of the smoothness of $F$. This approximation method has been successfully used to estimate probability measures in a number of applications (e.g., see [5], [3], [4], [7]).

Given the approximate distributions in (III.7), the relative permittivity is given by

$$
\widehat{\varepsilon}_{r 2}\left(\omega ; F^{N}\right)=\varepsilon_{\infty}-\sum_{k=1}^{N} \frac{\omega_{p} \alpha_{k}}{\omega^{2}-i \omega / \tau_{k}-\omega_{0}^{2}} .
$$

For all the simulations below, we choose $\mathcal{T}^{N}$ to be $N$ evenly spaced nodes over the interval $\left[1 \times 10^{-17}, 2 \times\right.$ $\left.10^{-14}\right]$, and the values for the parameters $\varepsilon_{s}$ and $\varepsilon_{\infty}$ are chosen as (adapted from [9])

$$
\varepsilon_{s}=2.25, \quad \varepsilon_{\infty}=1.0 .
$$

Preliminary data sets from available initial experiments involve excitation in the frequency range of $10^{13}$ to $10^{15}$ $\mathrm{Hz}$, because of this we choose a value of $\omega_{0}=1 \times 10^{14}$ as a baseline value for our computational examples.

Simulated Data: The measurement frequencies are taken at $\left\{\omega_{j}\right\}_{j=0}^{100}=\left\{10^{7+0.09 j}\right\}_{j=0}^{100}$. To generate the simulated data, the measurement error in (III.1) was chosen from a multivariate normal distribution with $\sigma_{0}=0.01$ or $\sigma_{0}=0.05$, and the true probability measure $F_{0}$ is chosen as $F_{0}=\alpha^{*} \Delta_{\tau_{1}^{*}}+\left(1-\alpha^{*}\right) \Delta_{\tau_{2}^{*}}$, where $\alpha^{*}=0.6, \tau_{1}^{*}=7.14 \times 10^{-16}$, and $\tau_{2}^{*}=1 \times 10^{-1^{2}}$. We note that in the case that the observed part of the system contains both the reflectance and the derivative of the reflectance, we need to calculate $\frac{\partial}{\partial \omega}\left|r_{s}\left(\omega_{j}, \theta, F\right)\right|$. However, a closed form analytical solution of this derivative has proved to be very difficult to obtain due to the form of $\left|r_{s}(\omega, \theta ; F)\right|$. Because of this, we take the forward difference to approximate the derivative; that is,

$$
\frac{\partial}{\partial \omega}\left|r_{s}\left(\omega_{j}, \theta, F\right)\right| \approx \frac{\left|r_{s}\left(\omega_{j+1}, \theta, F\right)\right|-\left|r_{s}\left(\omega_{j}, \theta, F\right)\right|}{\omega_{j+1}-\omega_{j}} .
$$

\section{NUMERICAL RESULTS}

We will use the above discussed delta approximation method to find an estimate $F^{N}$ for $F_{0}$, where the observable is either the reflectance or both the reflectance and the derivative of the reflectance.

\section{A. The Case Where the Observable is the Reflectance}

We first considered the case that only the reflectance can be observed (that is, $h$ is given by (III.2)). Figure 2 depicts the results obtained for the case with $\sigma=0.01$ using $N=30$ nodes in the approximation. We see from this figure that the model solution provides a good fit to the data, however the estimated distribution is not a good approximation to the true distribution. This indicates that the solution to the optimization problem is not unique (that is, the corresponding inverse problem is not well-posed). With an increased noise level $\sigma=$ 0.05 , as seen in Figure 3, the model solution begins to deviate from the data, and the estimated distribution is a poor approximation of the true distribution. Overall these two figures suggest that if only the reflectance can be observed, then we are unable to recover the true distribution even in the low noise level case (even though the fits to data are good).

\section{B. The Case Where the Observable is the Reflectance and the Derivative of the Reflectance}

We next considered the case where both the reflectance and the derivative of the reflectance can be observed (that is, $h$ is given by (III.3)). Figures 4 and 5 show the results obtained using $N=30$ nodes in the approximation for the cases with $\sigma_{0}=0.01$ and $\sigma_{0}=0.05$, respectively. We see from Figure 4 that the estimated reflectance and derivative of the reflectance are excellent fits to the true reflectance and derivative of the reflectance, and that the estimation for the distribution is very good. Figure 5 reveals that even in the case of higher noise levels the fits are still good and the estimated distribution is reasonably close to the true one. In addition, we found that the estimates are consistent across simulated data sets. Using the derivative information alone to estimate the probability measure was also considered. However, we found that this information 

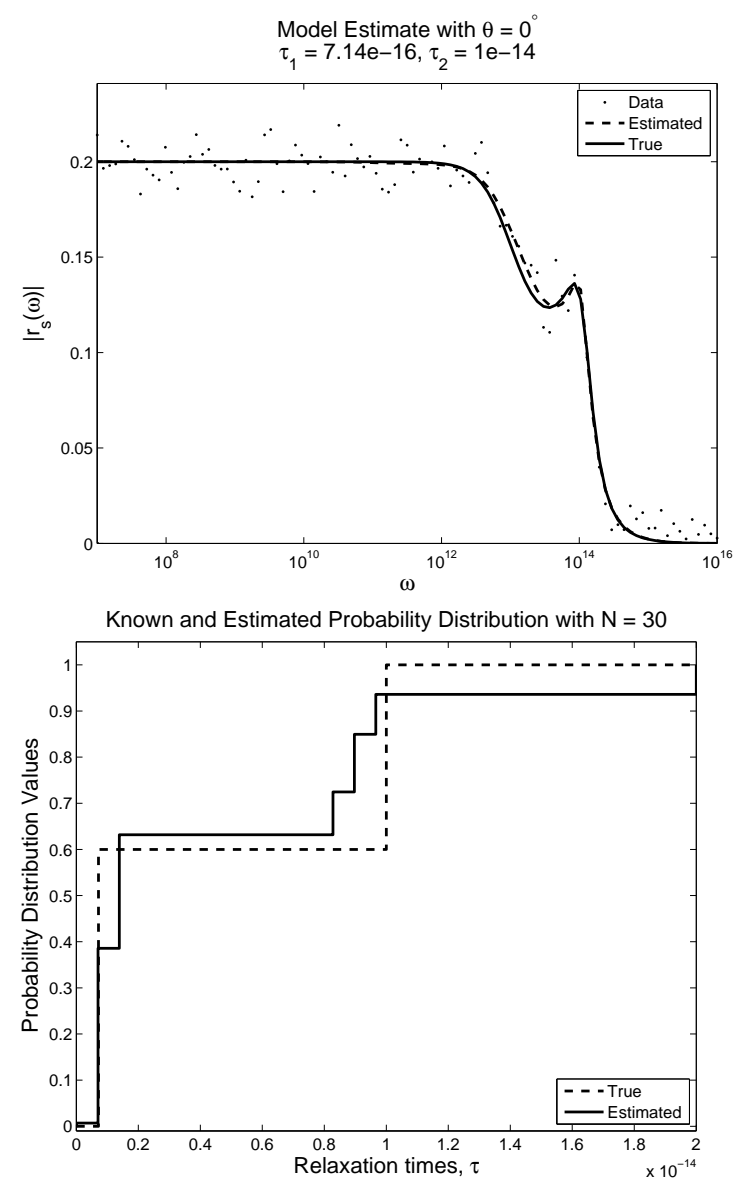

Fig. 2. The results obtained using the reflectance data with $\sigma_{0}=$ 0.01: (upper panel) the model fit to the data, and (lower panel) the true and estimated probability distributions.

is not enough to provide consistency in the estimation across simulated data sets.

\section{SUMMARY REMARKS}

In this note we use a Lorenz model as an example to investigate the type of data might be needed to accurately estimate a probability measure imposed on the model parameters through simulated data. The numerical results demonstrate that using the reflectance alone is not sufficient to recover the true distribution form even at low noise levels (even though the fits to the data are good). However, if both the reflectance and the derivative of the reflectance can be observed, then the estimated distribution is reasonably close to the true distribution even in the case where the noise level is relatively high. It is worth noting that the same conclusions were also reached when we tried to estimate the probability measure for the parameters in a Debye model. In addition, we also considered the case where the true distribution is some other type of distribution such as Gaussian or Bi-Gaussian, and we still obtained the same conclusions. We remark that the method used
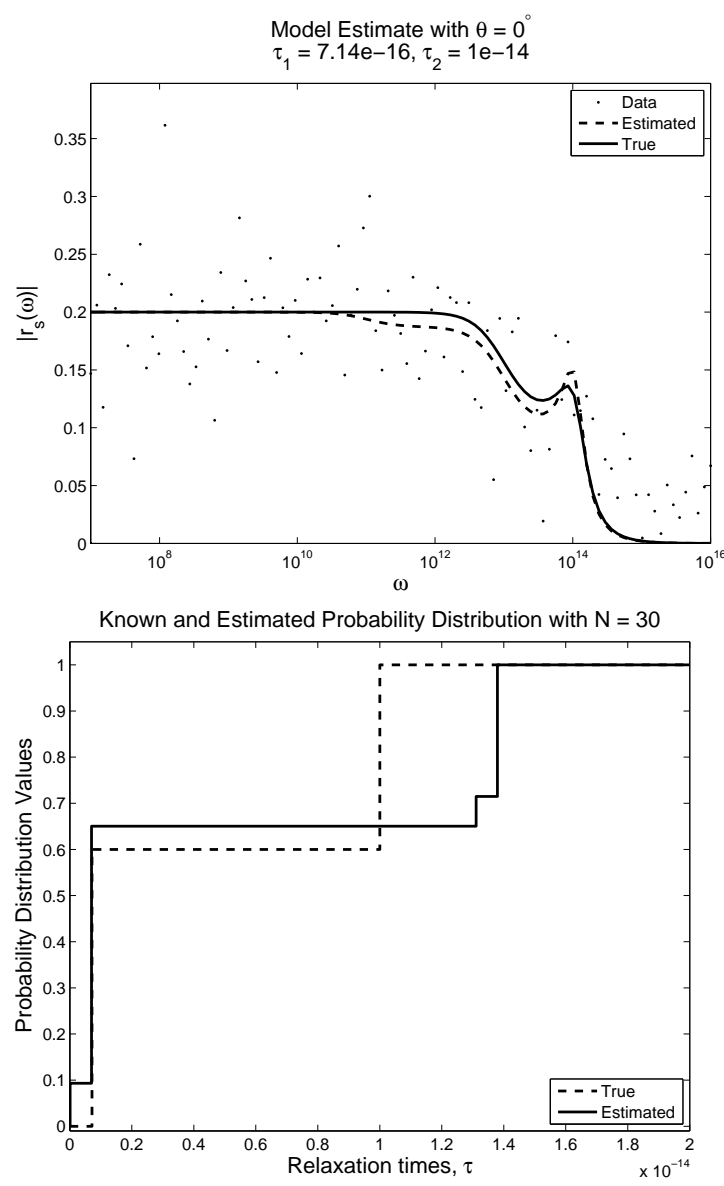

Fig. 3. The results obtained using the reflectance data with $\sigma_{0}=$ 0.05: (upper panel) the model fit to the data, and (lower panel) the true and estimated probability distributions.

to simulate the data for the derivative of the reflectance is somewhat artificial. However, we have preformed an important investigation and proof of concept which shows that if reliable derivative data can be obtained in practice, then one may be able to successfully determine the distribution of relaxation times.

\section{REFERENCES}

[1] H.T. Banks, A Functional Analysis Framework for Modeling, Estimation and Control in Science and Engineering, Chapman and Hall/CRC Press, Boca Raton, FL, 2012.

[2] H.T. Banks and K.L. Bihari, Modeling and estimating uncertainty in parameter estimation, Inverse Problems, 17 (2001), 95-111.

[3] H.T. Banks and B.G. Fitzpatrick, Estimation of growth rate distributions in size-structured population models, Quarterly of Applied Mathematics, 49 (1998), 215-235.

[4] H.T. Banks, B.G. Fitzpatrick, L.K. Potter and Y. Zhang, Estimation of probability distributions for individual parameters using aggregate population data, CRSC-TR98-6, January, 1998; In Stochastic Analysis, Control, Optimization and Applications, (Edited by W. McEneaney, G. Yin and Q. Zhang), Birkhäuser Verlag, Basel, 1998, 353-371.

[5] H.T. Banks and N.L. Gibson, Electromagnetic inverse problems involving distributions of dielectric mechanisms and parameters, Quarterly of Applied Mathematics, 64 (2006), 749-795. 

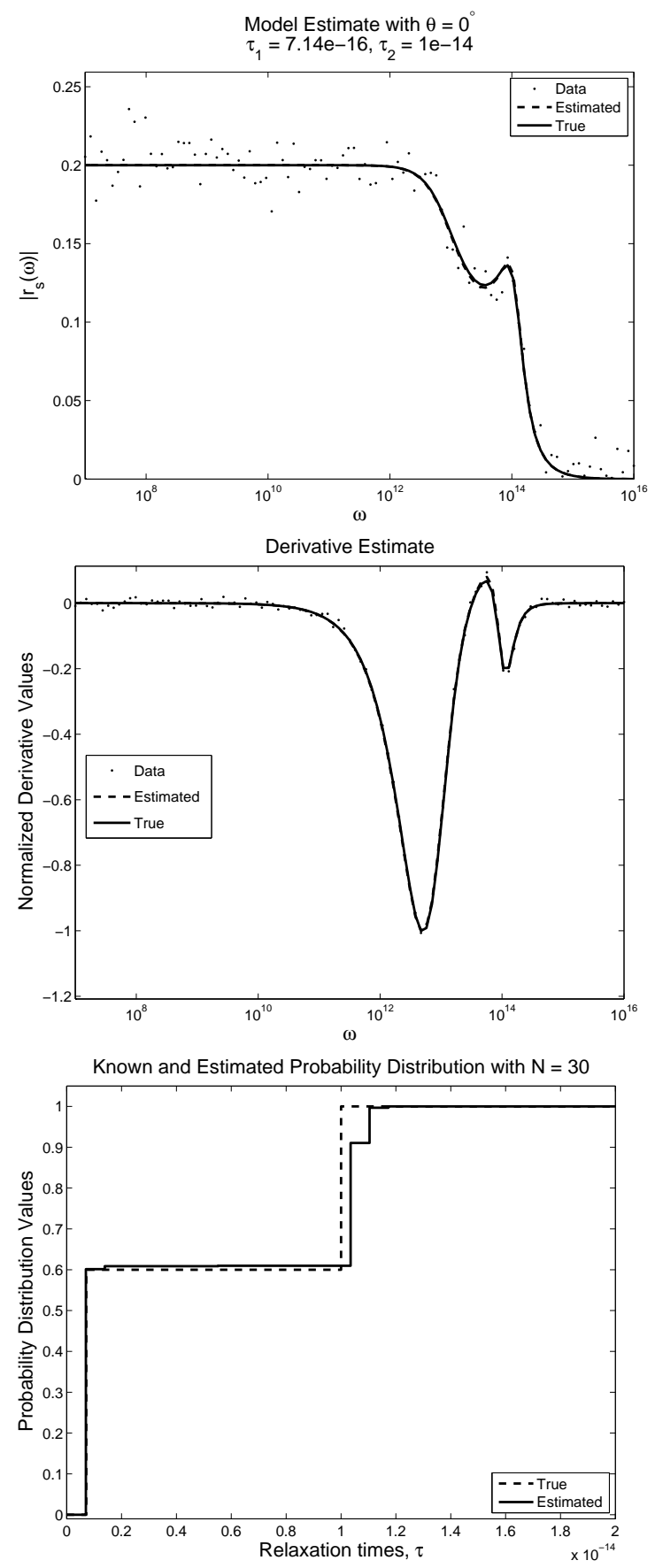

Fig. 4. Results obtained using both the reflectance and the derivative of reflectance data with $\sigma_{0}=0.01$ noise: (upper panel) The model fit to the data, (middle panel) the derivative fit to the derivative data, and (lower panel) the true and estimated probability distributions.

[6] H.T. Banks, Z.R. Kenz, and W.C. Thompson, A review of selected techniques in inverse problem nonparametric probability distribution estimation, J. Inverse and Ill-Posed Problems, 20 (2012), 429-460.

[7] H.T. Banks and G.A. Pinter, A probabilistic multiscale approach to hysteresis in shear wave propagation in biotissue, SIAM $J$. Multiscale Modeling and Simulation, 3 (2005), 395412.

[8] H.T. Banks and H.T. Tran, Mathematical and Experimental Modeling of Physical and Biological Processes, CRC Press,
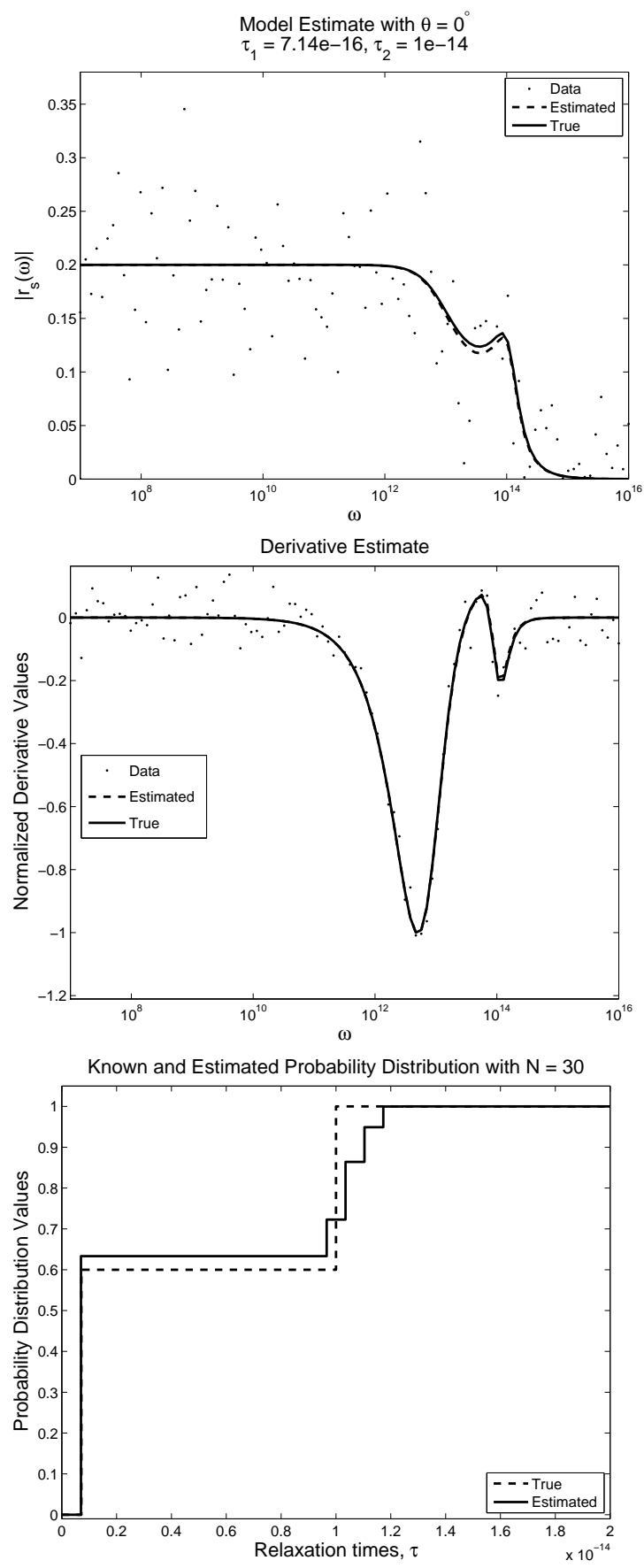

Fig. 5. Results obtained using both the reflectance and the derivative of reflectance data with $\sigma_{0}=0.05$ noise: (upper panel) The model fit to the data, (middle panel) the derivative fit to the derivative data, and (lower panel) the true and estimated probability distributions.

Boca Raton, FL, 2009.

[9] J.G. Blaschak and J. Fanzen, Precursor propagation in dispersive media from short-rise-time pulses at oblique incidence, Journal of the Optical Society of America A, 12 (1995), 1501-1512.

[10] Bruker OPUS spectroscopic software reference manual, version 4.2, 2003; ftp://ftp.gps.caltech.edu/pub/tccon/ tccon_manuals_and_documentation/IFS125/ OPUS_Manual.pdf.

[11] N.L. Gibson, Terahertz-Based Electromagnetic Interrogation 
Techniques for Damage Detection, PhD Thesis, NCSU, Raleigh, 2004.

[12] D.J. Griffiths, Introduction to Electrodynamics, 3rd edition, Prentice Hall, Upper Saddle River, New Jersey, 1999.

[13] Yu.V. Prohorov, Convergence of random processes and limit theorems in probability theory, Theor. Prob. Appl., 1 (1956), 157214.

[14] R.W. Schafer, What is a Savitzky-Golay filter? IEEE Signal Processing Magazine, (2011), 111-117. 